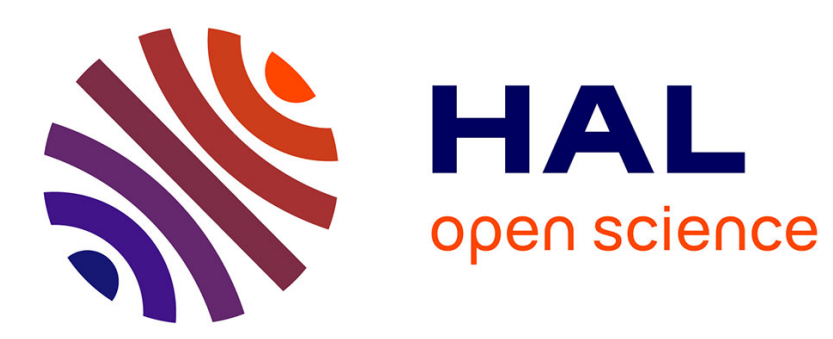

\title{
A novel nonsense PIEZO2 mutation in a family with scoliosis and proprioceptive defect
}

Marion Masingue, Julien Fauré, Guilhem Solé, Tanya Stojkovic, Sarah

Léonard-Louis

\section{- To cite this version:}

Marion Masingue, Julien Fauré, Guilhem Solé, Tanya Stojkovic, Sarah Léonard-Louis. A novel nonsense PIEZO2 mutation in a family with scoliosis and proprioceptive defect. Neuromuscular Disorders, 2019, 29, pp.75 - 79. 10.1016/j.nmd.2018.10.005 . hal-03486192

\section{HAL Id: hal-03486192 \\ https://hal.science/hal-03486192}

Submitted on 20 Dec 2021

HAL is a multi-disciplinary open access archive for the deposit and dissemination of scientific research documents, whether they are published or not. The documents may come from teaching and research institutions in France or abroad, or from public or private research centers.
L'archive ouverte pluridisciplinaire HAL, est destinée au dépôt et à la diffusion de documents scientifiques de niveau recherche, publiés ou non, émanant des établissements d'enseignement et de recherche français ou étrangers, des laboratoires publics ou privés.

\section{(c)(1) $\$$}

Distributed under a Creative Commons Attribution - NonCommercial| 4.0 International 


\section{A novel nonsense PIEZO2 mutation in a family with scoliosis and proprioceptive defect}

2 Marion Masingue ${ }^{1}$

3 Julien Fauré $e^{2,3}$

$4 \quad$ Guilhem Solé ${ }^{4}$

$5 \quad$ Tanya Stojkovic ${ }^{1}$

6 Sarah Léonard-Louis ${ }^{1}$

$7 \quad{ }^{1}$ Centre de Référence de Pathologie Neuromusculaire Nord/Est/Ile de France, Institut de

8 Myologie, CHU La Pitié-Salpêtrière, APHP, Paris, France

$9 \quad{ }^{2}$ University Grenoble Alpe F-38000 France

$10{ }^{3} \mathrm{CHU}$ Grenoble, Biochimie et Génétique Moléculaire, Grenoble, F-38000 France

$11{ }^{4}$ Centre de référence des maladies neuromusculaires AOC, Hôpital Pellegrin, CHU de

12 Bordeaux, Bordeaux, France

14 Corresponding author: Marion Masingue marion.masingue@aphp.fr 


\section{ABSTRACT}

PIEZO2 mutations have been described in dominant arthrogryposis, but homozygous mutations of PIEZO2 may also be responsible for more complex clinical patterns, associating distal arthrogryposis, neonatal respiratory insufficiency, scoliosis and proprioceptive impairment. We report here two sisters presenting with these clinical and genetic features. They had a similar phenotype, with severe hypotonia and respiratory distress at birth, delayed acquisition of motor milestones and need of scoliosis surgery. Hypotonia and alteration of proprioception were at the forefront of clinical examination for both, along with areflexia, hyperlaxity, cutis laxa, and discrete facial dysmorphy. Electrophysiological studies, including electroneuromyography and sensory evoked potentials, showed a mild sensory axonopathy without any myopathic features, but revealed a peripheral proximal lemniscal defect. Creatine kinase, muscular MRI and biopsy were normal, as well as cerebral MRI and neurometabolic biological explorations. They had a moderate restrictive syndrome on respiratory function tests and cardiac function was normal. Molecular studies performed on a panel of genes involved in distal arthrogryposis disclosed a nonsense homozygous c.3241C>T (p.Arg1051*) mutation in the PIEZO2 gene, which was also present at the heterozygous state in their mother's DNA. This new PIEZO2 mutation was in accordance with the phenotype combining arthrogryposis, scoliosis, hyperlaxity and proprioceptive impairment.

\section{HIGHLIGHTS}

Arthrogryposis and proprioceptive defects suggests a recessive PIEZO2 mutation Patients with arthrogryposis should be screened for PIEZO2 mutations 
38 Dominant PIEZO2 mutations are mostly missense whereas recessive cases are truncating

\section{KEYWORDS}

$40 \quad \mathrm{PIEZO} 2$

41 Arthrogryposis

42 Proprioceptive impairment

43

44 Abbreviations

45 ENMG: Electroneuromyography; SEP: Sensory Evoked Potentials; DA: Distal

46 Arthrogryposis; MWS: Marden-Walker Syndrome; 
48

49

Arthrogryposis is defined by reduced mobility of multiple joints and highly heterogeneous, with more than 400 genes described. Arthrogryposis can be of both dominant and recessive inheritance, and associated with other clinical features, namely orthopedic malformations, facial deformities, central nervous system (CNS) abnormalities, peripheral neuropathy or myopathy. [1] Among these genes, heterozygous missense PIEZO2 mutations have been associated with either distal Gordon Syndrome (Distal arthrogryposis (DA) type 3 or DA type 5 or Marden-Walker Syndrome (MWS) [5]. Later on, homozygous nonsense mutations in the PIEZO2 gene have been held responsible for arthrogryposis, scoliosis and, strikingly, proprioception defects [6], neonatal respiratory insufficiency [7, 8] and muscle weakness [9].

PIEZO polypeptides are mechanosensitive transmembrane cation channels [2,3]. They are expressed in several mechanosensitive tissues, such as neurons of the dorsal root ganglia, endothelium and visceral tissues, including lungs. Mouse models lacking PIEZO2 in mechanosensory neurons showed impaired limb coordination, and unstable gait [4].

We describe here the cases of two sisters prominently characterized by distal arthrogryposis and proprioception loss; a clinical phenotype which led us to find a novel nonsense homozygous mutation in the PIEZO2 gene.

\section{MATERIALS AND METHODS}

\subsection{Patients}

We report the cases of two twin sisters of Tunisian origin, followed from age 20 to 24 years (Patient II.3 and II.4), the first one (Patient II.3) at the Neuromuscular Reference Center of 
Pitié Salpêtrière Hospital (Paris) and the other (Patient II.4) at the Neuromuscular Reference Center in the University Hospital of Bordeaux.

\subsection{Clinical, electrophysiological and muscle Magnetic Resonance Imaging features}

Both sisters have undergone clinical examination, extended neurophysiological evaluations, including electroneuromyography (ENMG) and sensory evoked potentials (SEP). Routine biological analyses, including lysosomal enzymes and vitamin dosages, have been performed, as well as respiratory function tests, cardiac explorations (Patient II.3 and II.4), muscle Magnetic Resonance Imaging (MRI) (Patient II.3). and biopsy (Patient II.3 and II.4).

\subsection{Genetic studies}

All samples were processed after informed consents for molecular diagnosis had been obtained from the patients. Mutations in the probands' DNA have been screened on a panel of eleven genes involved in distal arthrogryposis. The design of the gene panel was realized with the AmpliSeq Designer tool (Thermo Fischer Scientific) and libraries for Next Generation Sequencing (NGS) were prepared according to manufacturer recommendations and analyzed on IonTorrent PGM instrument (Thermo Fischer Scientific). The coverage for the PIEZO2 gene (NM_022068) was $99.3 \%$ with a mean depth of 390X (ranging from 34 to 1486). Results of the sequencing were processed with the Torrent Suite 5.0 (Thermo Fischer Scientific) and annotation of the variants performed with a homemade script based on the Variant Effect Predictor tool [10]. The presence of the mutation was controlled by Sanger sequencing using specific primer designed to amplify exon 22 of the PIEZO2 gene and an ABI PRISM Big Dye Terminator Cycle Sequencing (V3.0) reaction kit with an analysis on an ABI 3130 DNA Analyzer (Thermo Fischer Scientific).

\section{RESULTS}

3.1.Clinical, electrophysiological and muscle MRI features 
Our 2 patients were born from healthy parents, who were not known to be consanguineous. They had 5 siblings, including one sister (II.2) who died at six years of age, with similar symptoms. Their father died in a car accident at age 32 (Figure 1)

Pregnancy was uneventful, but the 2 sisters were severely hypotonic at birth and experienced transient neonatal respiratory failure. Later on, they displayed delayed acquisitions of motor skills and started walking independently at 5 (Patient II.4) and 7 (Patient II.3) years only. From the onset of the disease, gait was very unstable, and a wheelchair was needed early on during childhood. They also had severe scoliosis, requiring arthrodesis in both cases.

There was no cognitive impairment, and both sisters were able to pursue higher studies.

On examination, at age 20 , hypotonia was still at the fore. Both patients displayed diffuse distal wasting along with muscular deficit and discrete fingers and wrist retractions, compatible with arthrogryposis. Hyperlaxity was noticed in multiple joints. Proprioceptive abnormalities were major, with diffuse apallesthesia, positive Romberg sign, and unsteady wide-based gait, as well as dysmetria appearing with eyes closed. Tendon reflexes were abolished in the four limbs. There was no facial involvement, no cerebellar ataxia, no pyramidal or bulbar syndrome, and no ocular motor impairment. Patient II.3 also displayed cutis laxa (Figure 2)

ENMG showed a mild sensory axonal neuropathy in patient II.3 and was normal in patient II.4. There was no myopathic feature in both sisters. SEP revealed bilateral impairment of lemniscal sensory pathways for both (Figure 3). Cerebral and medullar MRI were normal, apart from the scoliosis. Muscular MRI showed no abnormality in patient II.3. Muscular biopsy was normal for patient II.3 and showed mild muscular abnormalities for patient II.4: type 2 fiber predominance and rare isolated concentric lamellar bodies. 
Respiratory function tests revealed a mild restrictive syndrome. The forced vital capacity was 46\% (Patient II.3) and 69\% (Patient II.4) of predicted value. No other functional studies were realized to assess diaphragmatic function.

Echocardiography and electrocardiogram were normal.

\subsection{Molecular findings}

Patient 1 was first screened for the FKBPI4 gene, known to be responsible for Ehlers-Danlos Syndrome with progressive kyphoscoliosis and myopathy [11]. No mutation was found, and DNA of the patient was then screened for genes involved in distal arthrogryposis on a gene panel with an IonTorrent PGM instrument. The mean coverage for a minimal depth of $30 \mathrm{X}$ for the 11 genes explored was $92.45 \%$. The analysis of SNP with an allelic frequency under a threshold of $0.5 \%$ in the EXAC database identified only the c.3241C>T;p.Arg $1051 *$ variation in the exon 22 of the PIEZO2 gene at the homozygous state. This variation resulted in a premature STOP codon in exon 22 and in a biallelic loss-of-function of the PIEZO2 gene. The segregation of the variation performed on the DNA of the probands' mother and sister showed compatibility with a recessive mode on inheritance. The father's DNA was not available.

\section{DISCUSSION}

A recessive condition associating arthrogryposis and a proprioception defect with transient respiratory failure, scoliosis, hypotonia, hyperlaxity is highly evocative of PIEZO2 biallelic loss-of-function mutation, as established by previously published works [6-9] (Table 1). The clinical phenotype of our two patients, encompassing these features, led us to a specific DNA sequencing targeting the PIEZO2 gene, and allowed us to identify a novel homozygous mutation, without using whole exome or mendeliome sequencing as previously described [6- 
9]. It is worth noticing that our two patients, although most of our symptoms were similar, displayed some phenotypical differences: age of motor skills achievement, ENMG and biopsy findings. The fact that they were followed in two different centers could account for those findings, this might suggest that there is some variability in the clinical expression of the disease.

\subsection{Two types of PIEZO2 mutations inducing two different phenotypes}

PIEZO2 gene mutations are particularly interesting and may cause two very distinguishable phenotypes according to the type of mutation and their mode of inheritance. Homozygous recessive mutations lead to a loss of function of the gene, whereas heterozygous dominant mutations cause a gain of function. Consequently, those two mechanisms induce very different conditions: although they share some clinical characteristics, namely arthrogryposis and scoliosis, there are distinguished by the presence of severe alterations of proprioception in the biallelic PIEZO2 whereas the heterozygous mutations correspond to a purer form of distal arthrogryposis, such as Gordon Syndrome, Marden-Walker Syndrome, or Distal Arthrogryposis Type $5[2,5]$.

\subsection{PIEZO2 and proprioception}

So far, mutations of PIEZO2 seem to be the only possible genetic cause of proprioception impairment with arthrogryposis. In humans, specialized mechanosensory neurons called proprioceptors convey information about the stretch and tension experienced by muscles, tendons, skin, and joints. PIEZO polypeptides are mechanosensitive cation channels and are activated in response to mechanical displacement of the cell surface membrane. [3]

PIEZO2 is expressed, in particular, at the endings of somatosensory neurons, which convey information about tension and stretch experienced by joints, skin, muscles and tendons $[4,8]$. Mice models have evidenced that PIEZO2 is responsible for the transduction of rapidly- 
adapting mechanically-activated currents in the neurons of the dorsal root ganglia [2]. These findings explain why PIEZO2 loss-of-function mutations cause a very specific phenotype with a characteristic proprioceptive defect

The mild axonal neuropathy observed in Patient II.3 seems to be a frequent characteristic of patients with biallelic PIEZO2 mutations [7-9], although not explaining the proprioceptive defect in itself.

4.3. Possible mechanisms for other clinical features encountered in PIEZO2 mutations

It has been suggested that PIEZO2 mutations may cause dysregulation of mechanotransduction affecting both cartilage constitution [12] and development of skeletal muscles [5] possibly leading to arthrogryposis and orthopedic abnormalities.

As a matter of fact, in patient 2, muscle biopsy revealed non-specific mild myopathic features, findings that have been described previously in recessive PIEZO2 mutations [9].

Respiratory impairment has also been described and is at least partially explained in adulthood by severe scoliosis and diaphragmatic weakness, whereas in cases of neonatal respiratory failure, it is more likely the consequence of diaphragmatic weakness only. Alternatively, a role for PIEZO2 was suggested as a mechanosensor required for correct lung development [13].

4.4.A unique cause for proprioceptive defects and arthrogryposis

Hereditary proprioceptive defect is caused by numerous hereditary sensory neuropathies but usually with severe axonal neuropathy. It has much more rarely been described when caused by proximal lemniscal pathways impairment. Neurometabolic diseases such as Friedreich ataxia, Vitamin E deficiency or hypobetalipoproteinemia may show posterior cord involvement on SEPs, but patients also display central nervous system symptoms $[14,15]$. A 
case of hereditary sensory ataxic neuropathy has been reported in a Japanese family with a mild sensory axonal neuropathy but significantly prolonged central conduction time on SEPs. Patients were not screened for PIEZO2 mutations, but, unlike ours, their sensory complaints went beyond sole ataxia, proximal muscle weakness was systematic, age at onset was much older (after 40 years), and there was neither scoliosis nor respiratory failure. [16].

More recently, mutations in RFN170 coding for the Ring Finger Protein 170 have been described, responsible for progressive sensory ataxia linked to degeneration of the posterior columns of the spinal cord. Patients with RFN170 mutations had, however, a progressive, isolated, form of ataxia predominating in the legs; they were older, and inheritance was dominant. [17]. Mutations in the FLVCRI gene also cause posterior column ataxia, sometimes associated with scoliosis and retinis pigmentosa,, but ENMG reveals a ganglionopathy [18].

Finally, secondary arthrogtyposis may occur in several neuromuscular disorders, and thus could be associated with conditions causing proprioceptive defects and neuropathy. However, in those cases, it tends to appear later on in the course of the disease than in our patients.

PIEZO2 is a unique gene with two very distinct phenotypes corresponding to the inheritance mode. As heterozygous dominant mutations are responsible for arthrogryposis multiplex congenita, PIEZO2 biallelic mutations have a typical clinical presentation, with scoliosis and proprioceptive deficit as main symptoms, together with arthrogryposis, hypotonia and transient neonatal respiratory failure. The role of PIEZO2 as a mechanosensitive receptor in somatosensory neurons and its implication in bones and joint development as well as lung development probably intervene in the occurrence of those symptoms. The association of these clinical features is highly evocative and should prompt practitioners to screen PIEZO2 
209 gene, as few other disorders display the same characteristics.

210

211 
DECLARATIONS

213

214

215

216

217

218

219

220

221

222

223

224

225

Ethics approval and consent to participate: Authors declare that this work received all necessary ethical approvals and that all the patients involved consented to participate.

\section{Consent for publication: Yes.}

Availability of data and material: Please contact author for data requests.

Competing interests: Authors declare no financial or non-financial competing interests in relation to this manuscript.

Funding: No funding was necessary

Author contributions: MM, JF, GS, TS, SLL were involved in conception and design of the research project, in analysis and interpretation of the data. MM, TS and SLL were involved and writing of the first draft of the manuscript. SLL and GS were involved in the acquisition of clinical data and JF was involved in the acquisition of molecular data. All authors read and approved the final manuscript. 


\section{REFERENCES}

228

229

230

231

232

233

234

235

236

237

[1] Hall, JG. Arthrogryposis (multiple congenital contractures): diagnostic approach to etiology, classification, genetics, and general principles. Eur J Med Genet. 2014;57(8):464-72

[2] Coste B, Mathur J, Schmidt M, Earley TJ, Ranade S, Petrus Mj, et al. Piezo1 and Piezo2 are essential components of distinct mechanically-activated cation channels. Science. 2010 October $1 ; 330(6000):$ 55-60.

[3] Alper SL. Genetic Diseases of PIEZO1 and PIEZO2 dysfunction. Curr Top Membr. 2017;79:97-134

[4] Woo SH, Lukacs V, De Nooij JC, Zaytseva D, Criddle CR, Francisco A, et al. Piezo2 is the principal mechanotransduction channel for proprioception. Nat Neurosci. $2015 ; 18(12): 1756-62$

[5] McMillin, M. J., Beck, A. E., Chong, J. X., Shively, K. M., Buckingham, K. J., Gildersleeve, H. I et al. Mutations in PIEZO2 cause Gordon syndrome, Marden-Walker syndrome, and distal arthrogryposis type 5. Am J of Hum Gen. 2014;94(5):734-44.

[6] Mahmud AA, Nahid NA, Nassif C, Sayeed MSB, Ahmed MU, Parvee M, et al. Loss of the proprioception and touch sensation channel PIEZO2 in siblings with a progressive form of contractures. Clin Genet. 2017;91(3):470-75

[7] Delle Vedove A, Storberck M, Heller R, Hölker I, Hebbar M, Shukla A, et al. Biallelic loss of proprioception-related PIEZO2 causes muscular atrophy with perinatal respiratory distress, arthrogryposis and scoliosis. Am J of Hum Gen. 2016;99(5):1206-16

[8] Chelser AT, Szczot M, Bharucha-Goebel D, Ceko M, Donkervoort S, Laubacher C et al. The role of PIEZO2 in Human Mechanosensation. N Engl J Med. 2016:375(14):1355-64 
249 [9] Haligolu G, Becker K, Temucin C, Talim B, Kuçukçahin N, Pergande M, et al. Recessive

250 PIEZO2 stop mutation causes distal arthrogryposis with distal muscle weakness, scoliosis and

251 proprioception defects. J Hum Genet. 2017;62(4):497-501

252 [10] McLaren W, Gil L, Hunt SE, Riat HS, Ritchie GR, Thormann A, et al. The Ensembl

253 Variant Effect Predictor. Genome Biol. 2016:17:122

254 [11] Baumann M, Giunta C, Krabichler B, Rüschendorf F, Zoppi N, Colombi M et al.

255 Mutations in FKBP14 cause a variant of Ehlers-Danlos syndrome with progressive

256 kyphoscoliosis, myopathy, and hearing loss. Am J Hum Genet. 2012;10;90(2):201-16.

257 [12] Lee W, Leddy HA, Chen Y, Hee Lee S, Zelenski A, McNulty Al et al. Synergy between

258 Piezo1 and Piezo2 channels confers high-strain mechanosensitivity to articular cartilage. Proc

259 Natl Acad Sci U S A. 2014;111(47):E5114-22

260 [13] Nonomura K, Woo SH, Chang RB, Gillich A, Qiu Z, Francisco AG. Piezo2 senses

261 airway stretch and mediates lung inflation-induced apnoea. Nature. 2017;541(7636):176-81.

262 [14] Tsuji S, Uozomi T, Ito Y, Ohnishi A, Murai Y. Serial somatosensory evoked potentials

263 in a patient with familial hypobetaliproteinemia and vitamin E deficiency. Jpn J Med

$264 \quad 1990 ; 29(6) 628-32$

265 [15] Zouari M, Feki M, Ben Hamida C, Larmaout A, Turki I, Belal S et al. Electrophysiology

266 and nerve biopsy: comparative study in Friedreich's ataxia and Friedreich's ataxia phenotype

267 with vitamin E deficiency. Neuromuscul Disord. 1998;8(6):416-25

268 [16] Murakami T, Fukai Y, Rikimaru M, Henmi S, Ohsawa Y, Sunada Y. Hereditary sensory

269 ataxic neuropathy associated with proximal muscle weakness in the lower extremities. J

$270 \quad$ Neurol Sci. 2010;291(1-2)121-3 
271 [17] Valdmanis PN, Dupré N, Lachance M, Stochmanski SJ, Belzil VV, Dion PA et al. A

272 mutation in the RNF170 gene causes autosomal dominant sensory ataxia.Brain. 2010;134(Pt

$273 \quad 2): 602-7$

274 [18] Rajadhyaksha AM, Elemento O, Puffenberger EG, Schierberl KC, Xiang JZ, Putorti ML

275 et al. Mutations in FLVCR1 cause posterior column ataxia and retinitis pigmentosa. Am J

276 Hum Genet. 2010;87(5):643-54

277

278 
279 Table 1: Genetic and clinical characteristics of previously published recessive mutations in

280 the PIEZO2 Gene

281

282 Figure 1: Family pedigree

283

284

285

286

287

288

289

290

291

292

293

294

295

296

Figure 2: Patients clinical features

Both patients displayed distal wasting and arthrogryposis (A and D), hyperlaxity (B) and severe scoliosis (C).

Figure 3: Sensory Evoked Potentials in the upper limbs of Patient 1

Peripheric conduction was normal (N5). N13 amplitude was reduced on the left upper limb (A, white circle) and absent on the right (B, white arrow). Bulbar components (N/P14 - black circle) were reduced and latency of cortical components (N9-N20) were extended, indicating bilateral impairment of the lemniscal pathways. 

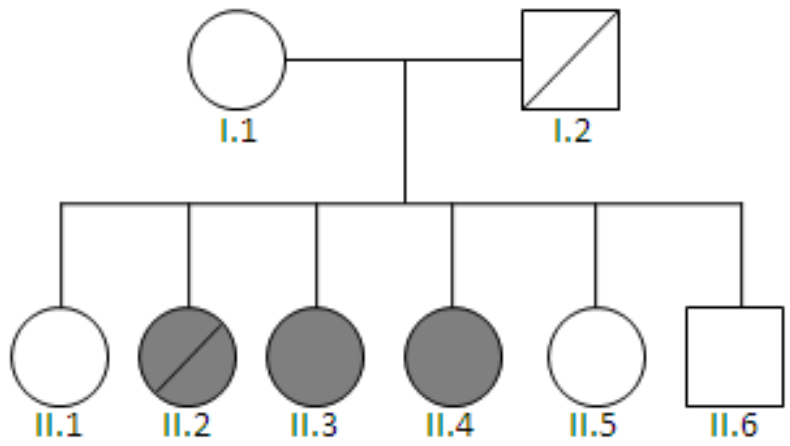


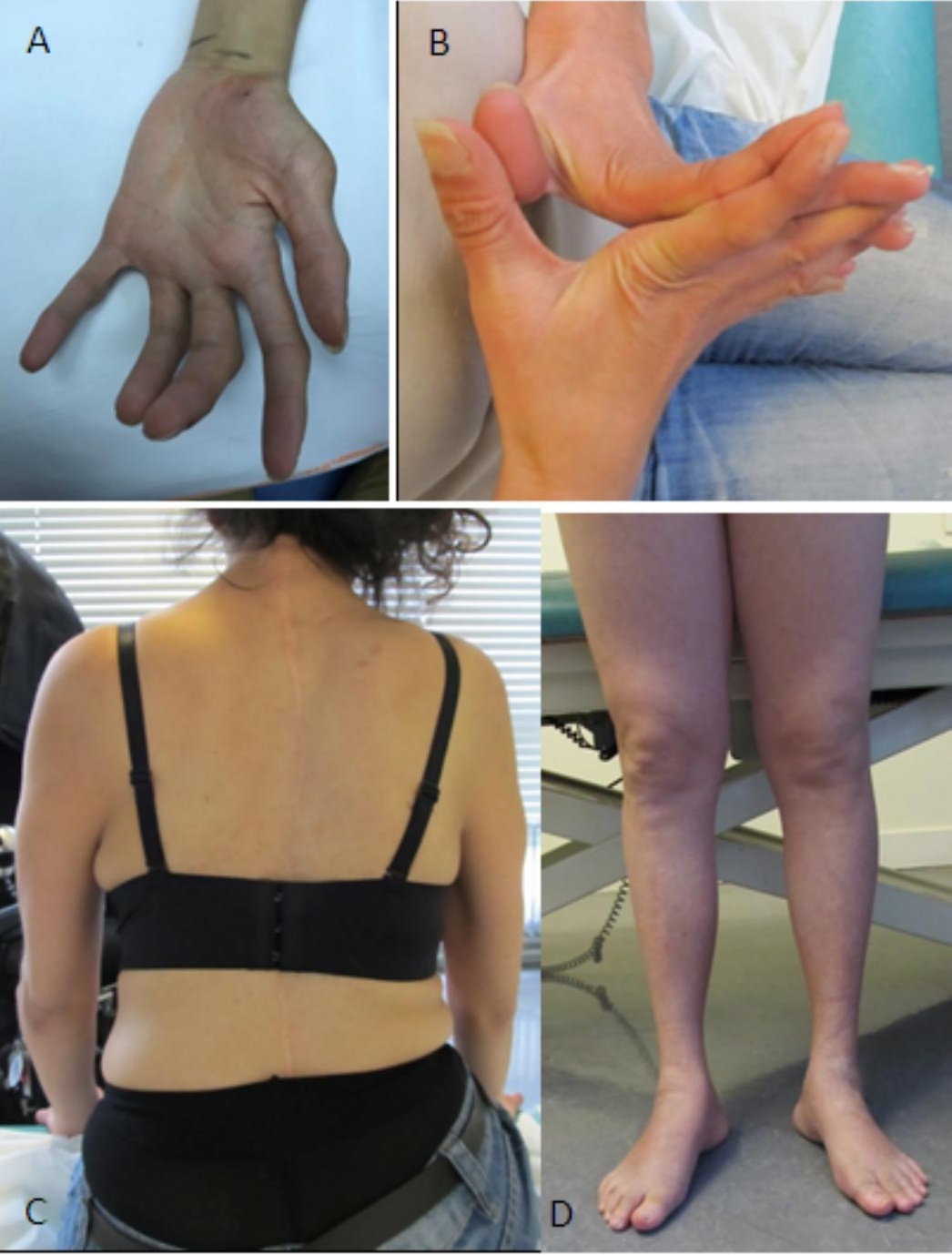


A

$\mathrm{VCN}$

$1 \mathrm{n} ı \mathrm{~V} / \mathrm{n} 5 \mathrm{mc} / \mathrm{n}$

Erb's-Fz

5ıI/n $5 \mathrm{me} / \mathrm{n}$

C7-Cou

5ıv/n 5me/n

$\mathrm{C} 7-\mathrm{Fz}$

5ı1/n $5 \mathrm{~ms} / \mathrm{n}$

ParD-ErbD

5 IIV/n $5 \mathrm{ma} / \mathrm{n}$

Cz-Erbcontra

5 IIV/n $5 \mathrm{me} / \mathrm{n}$

ParG-ErbD

$5 ı \mathrm{v} / \mathrm{n} 5 \mathrm{mc} / \mathrm{n}$

PaD-PaG

$5 \Perp v / n$ ma/n

B

VCN

10 1I/n $5 \mathrm{me} / \mathrm{n}$

Erb's-Fz

5 IIV/n $5 \mathrm{me} / \mathrm{n}$

C7-Cou

5 IV/n $5 \mathrm{me} / \mathrm{n}$

C7-Fz

$5 ı \mathrm{~V} / \mathrm{n}$ 5me/n

ParG-ErbG

$5 ı \mathrm{~V} / \mathrm{n} 5 \mathrm{me} / \mathrm{n}$

Cz-Erbcontra

5ıv/n 5ma/n

ParD-ErbG

$5 ı \mathrm{~V} / \mathrm{n} 5 \mathrm{me} / \mathrm{n}$

PaG-PaD

5 IIV/n $5 \mathrm{me} / \mathrm{n}$

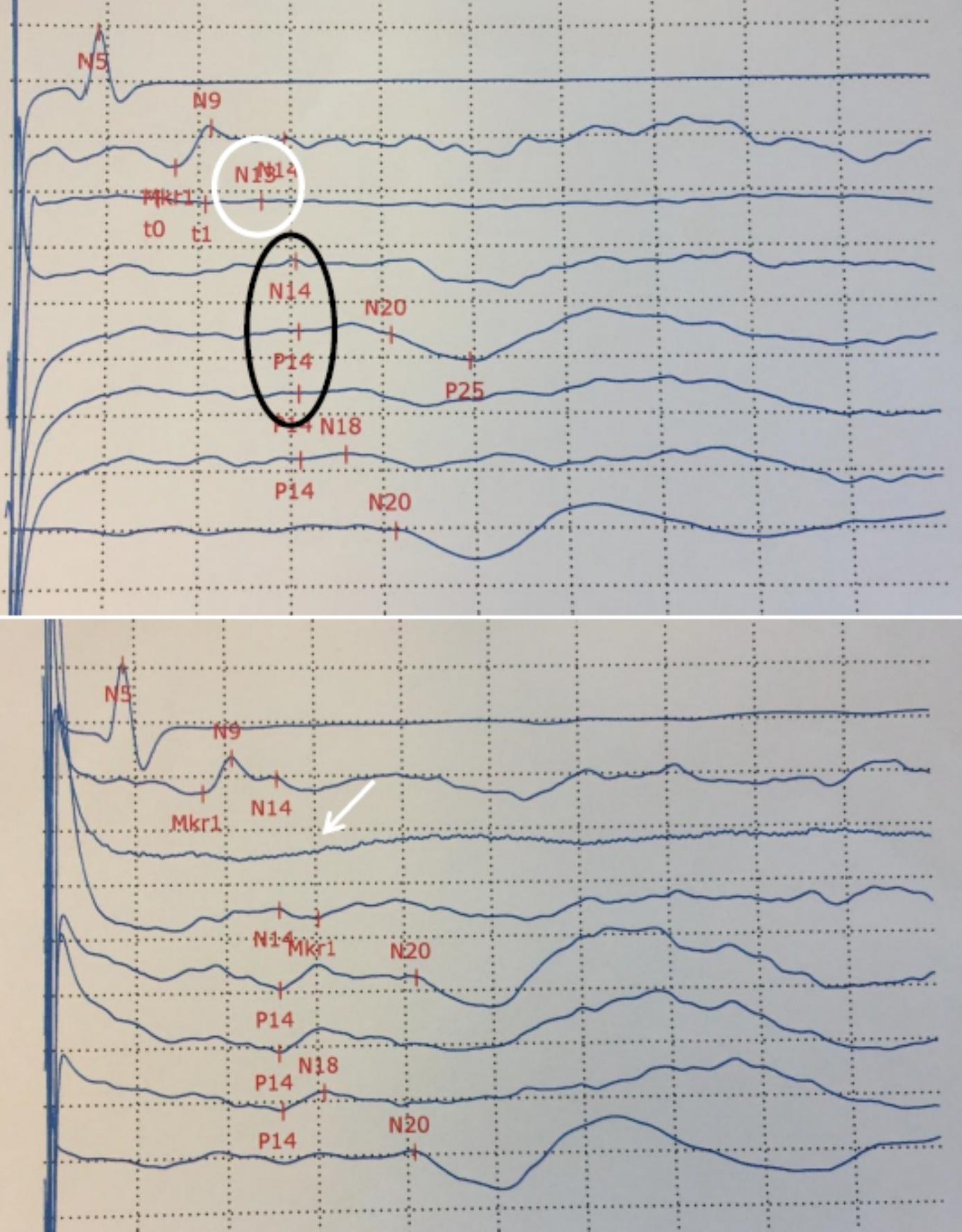




\begin{tabular}{|c|c|c|}
\hline & Clinical Features & Mutation \\
\hline $\begin{array}{l}\text { Delle Vedove } \\
\text { et al. } 2016\end{array}$ & $\begin{array}{l}10 \text { individuals, } 4 \text { families } \\
\text { Arthrogryposis, spontaneously } \\
\text { resolving respiratory insufficiency } \\
\text { at birth, muscular atrophy > distal } \\
\text { lower limbs, scoliosis, mild sensory } \\
\text { involvement }\end{array}$ & $\begin{array}{l}\text { - c.5621delT (p.Leu1874Argfs*5) homozygous } \\
\text { - c.3019_3029del(p.Pro1007Leufs*3) homozygous } \\
\text { - c.1550_1552delGCTinsCGAA (p.Ser517Thrfs*48) } \\
\text { homozygous } \\
\text { - c.493-?_917+?del (p0?) homozygous }\end{array}$ \\
\hline $\begin{array}{c}\text { Chelser et al. } \\
2016\end{array}$ & $\begin{array}{l}2 \text { subjects, } 2 \text { families } \\
\text { Scoliosis, hypotonia and shallow } \\
\text { breathing during infancy, unsteady } \\
\text { gait with proprioceptive defect }\end{array}$ & $\begin{array}{l}\text { - c.4723>T (p.R1575*)/ c.5053C>T (p.R1685*) } \\
\text { - c.5054G >C (p.R1685P)/ c.5053C>T (p.R1685*) }\end{array}$ \\
\hline $\begin{array}{l}\text { Haligolu et } \\
\text { al. } 2017\end{array}$ & $\begin{array}{l}\text { Hypotonia, distal laxity, } \\
\text { contractures, muscle weakness, } \\
\text { scoliosis, proprioceptive defect }\end{array}$ & c.1384C>T, (p.R462*) homozygous \\
\hline $\begin{array}{l}\text { Mahmud et } \\
\text { al. } 2017\end{array}$ & $\begin{array}{l}\text { Scoliosis, contractures involving } \\
\text { distal joints, proprioception and } \\
\text { touch sensation defects }\end{array}$ & c.2708C >G; p.S903* homozygous \\
\hline
\end{tabular}

